\title{
ACCURACY OF A LOW-COST NOVEL COMPUTER-VISION DYNAMIC MOVEMENT ASSESSMENT: POTENTIAL LIMITATIONS AND FUTURE DIRECTIONS
}

\author{
Mark McGroarty $^{1 *}$, Susan Giblin ${ }^{1}$, Dara Meldrum ${ }^{2}$, Friedrich Wetterling ${ }^{1}$ \\ ${ }^{1}$ Kitman Labs Ltd., Joyce's Walk, Dublin 1, Ireland \\ ${ }^{2}$ School of Physiotherapy, Royal College of Surgeons, Dublin, Ireland \\ *corresponding author: Mark McGroarty ( markmcgroarty@kitmanlabs.com )
}

KEY WORDS: Low Cost, Markerless, Motion Capture, Movement Assessment, ACL.

\begin{abstract}
:
The aim of the study was to perform a preliminary validation of a low cost markerless motion capture system (CAPTURE) against an industry gold standard (Vicon). Measurements of knee valgus and flexion during the performance of a countermovement jump (CMJ) between CAPTURE and Vicon were compared. After correction algorithms were applied to the raw CAPTURE data acceptable levels of accuracy and precision were achieved. The knee flexion angle measured for three trials using Capture deviated by $-3.8^{\circ} \pm 3^{\circ}$ (left) and $1.7^{\circ} \pm 2.8^{\circ}$ (right) compared to Vicon. The findings suggest that low-cost markerless motion capture has potential to provide an objective method for assessing lower limb jump and landing mechanics in an applied sports setting. Furthermore, the outcome of the study warrants the need for future research to examine more fully the potential implications of the use of low-cost markerless motion capture in the evaluation of dynamic movement for injury prevention.
\end{abstract}

\section{INTRODUCTION}

\subsection{General Instructions}

A tear of the anterior cruciate ligament (ACL) is one of the most sever career debilitating injuries that can cause long term absence from sport and can lead to osteoarthritis in later years (Paterno et al. 2010). The ACL is one of three cruciate ligaments which serves to stabilise the knee joint, preventing excessive translation of the tibia relative to the femur. ACL injuries can occur in a contact or noncontact manner in high intensity intermittent sports that involve rapid changes of direction. Noncontact ACL injuries account for $70 \%$ to $84 \%$ of all ACL tears in both female and male athletes (McNair et al., 1990; Boden et al. 2000; Faunø and Wulff Jakobsen 2006). ACL injuries are found to occur after initial contact in landing or cutting manoeuvres with the knee at full extension (Krosshaug et al. 2007). Risk factors for ACL occurrence include dynamic re-stablisation on jump landing and poor dynamic co-ordination during eccentric loading jump phases. For example, asymmetries between knee and hip mechanics during jump movement indicate risk of ACL (Renstrom et al. 2008). However, measures of knee and hip joint function discretely, or indeed measuring performance outcome (jump height or force production) in isolation, fails to provide quantitative indicators on these essential precursors to injury (i.e. kinetic chain coordination and asymmetry (Renstrom et al. 2008)). Appropriate pre-screening procedures could determine the parameters that put athletes at an increased risk of ACL injury.

The countermovement jump (CMJ) is a well-researched, popular screen used to track athlete power and force production (Cormie, McBride, and McCaulley 2009). In addition to typical performance outcomes, the CMJ involves complex kinematics that places demands on neuromuscular and neurocognitive systems (Swanik et al. 2007). Previous research by Dufek and Bates supports a link between landing forces and knee injury (Dufek and Bates 1991). Oñate et al. (2005) support the link between landing kinematics and kinetics, thus suggesting that the subsequent load placed on ACL from landing kinetics and kinematics are interlinked in a multiplanar manner (Oñate et al.
2005). There is limited evidence in the literature on current prospective biomechanical risk factors for ACL injury including monitoring methods (Padua et al. 2015), research on ACL injury mechanisms has begun to identify and evaluate kinematic factors proximal to the knee joint (Blackburn and Padua 2008). It has been suggested that hip internal rotation and adduction contributes to knee valgus immediately following landing (McLean, Lipfert, and van den Bogert 2004; Zeller et al. 2003). The measurement of knee valgus motion from initial contact to peak flexion, frontal plane knee angle at initial contact, frontal plane knee angle at peak flexion, and knee-to-ankle separation ratio at peak flexion, have proven to be useful in screening for future ACL injury (Stone et al. 2013). The objective analysis of landing mechanics and in particular knee valgus is paramount in the identification of risk factors for future ACL injury (Hewett et al. 2005b).

In order to quantify and analyse the factors that contribute to ACL injury risk, expensive equipment such as 3D motion capture systems have been utilised to provide quantitative biomechanical measurements that link to risk of injury in professional athletes (Hewett et al. 2005a). The current gold standard motion capture system for joint location during dynamic movements is the Vicon system. It relies on markers being placed in well-defined anatomical locations on the athlete under investigation. Several clinically-based monitoring methods included the use of 3-D motion capture equipment and force plates (Ford et al. 2005; Chappell et al. 2002; Ford, Myer, and Hewett 2003). While VICON can be used in a clinical setting, its application as a high throughput monitoring tool for athletes is impractical. This highlights the need for the development of an accurate, low-cost, and markerless scanner that can facilitate large-scale field based screenings.

Recent studies (Stone et al. 2013; Bonnechère et al. 2013) evaluated the reliability of the Microsoft Kinect in capturing biomechanical measures. Bonnechère et al. compared the Kinect to a marker based system and found large deviations from the expected measurements when subjects were performing squat movement (Stone et al. 2013; Bonnechère et al. 2013). Although the output of Kinect is deemed to be insufficiently accurate for clinical measurements, sophisticated 
software solutions were suggested as viable solutions to improve the measurement accuracy in order to satisfy clinical standards (Pfister et al. 2014). The aim of this paper is to explore the accuracy and precision limits of a state-of-the-art biomechanics software package (Kitman Labs Ltd., Dublin, Ireland) which refines skeletal information acquired by the Microsoft Kinect. This is achieved in a comparative study measuring the accuracy and reliability of Kitman Labs Biomechanics solution against the marker based system Vicon during the performance of three countermovement jumps (CMJ).

\section{METHODS}

2.1 Participants: Data was obtained from a single healthy athletic male participant (age $=26$ years, height $=176 \mathrm{~cm}$, weight $=82 \mathrm{~kg}$ ). The participant had no current injury or injury history that was impeding participation in sport. In accordance with ethical procedures, informed consent was obtained. A Physical Activity Readiness Questionnaire (PAR-Q) was completed prior to task completion.

2.2 Measurement System: A single-case comparison was completed between Vicon and Capture. A 6-camera Vicon system (sampling at $100 \mathrm{~Hz}$ ) was positioned in a circular fashion around the athlete so that all body segments were visible enabling 3D reconstruction. Retro-reflective markers were placed on the head $(n=4)$, trunk $(n=5)$, upper limbs $(n=14)$, pelvis $(n=4)$ and lower limbs $(n=12)$. The markers tracked multiple body segments in the sagittal, coronal and transverse planes using the Vicon's 2.1 Nexus and Plug-in-Gait software modelling systems. The raw data was exported to Matlab/Visual 3D for processing. Prior to testing, a calibration procedure was used to define the 3D testing volume using Vicon's software (NEXUS) and standard operating procedures (Vicon Motion Systems Ltd., Oxford, UK).

2.3 Test Procedure: Standardised instruction was provided to the participant and a number of familiarisation (sub maximal) jumps were completed to ensure proper technique and to account for a learning effect. A physiotherapist experienced in using Vicon captured and processed all Vicon data. Reliability had previously been established in the lab (Meldrum et al 2013).

2.4 Kitman Labs biomechanics scanning solution: Kitman Labs Ltd. provides an advanced software solution to acquire skeletal information using Kinect version 2 sensor (Kinect for Windows and Xbox One, Microsoft corporation, Redmond, WA, USA) and Software Developer Kit (SDK) via its graphical user interface - CAPTURE. In CAPTURE the athlete is first directed to move into the initial position in front of the sensor ensuring that the full $\mathrm{CMJ}$ can be recorded for any athlete height. In a second step, calibration data of the limb lengths are recorded during a $1 \mathrm{~s}$ calibration phase while the athlete is instructed to stand still in neutral stance. In a third step, the start of the CMJ acquisition is indicated on the CAPTURE screen. In a last step, the data is checked for quality measures and the data is securely transferred to the Kitman Labs server. All further handling of the data, display of results, and alerting is facilitated in PROFILER - the Kitman Labs data base and athlete management system. The raw data is processed to correct for limb length variations using the calibration information. A coordinate transformation is used to convert the joint centers data from Kinect space to real world coordinate space. The jump phases were derived using the time of peak loading (pre jump phase) and stabilisation (post jump phase) as well as the normalised head height during the squat. The range of pre jump phase was determined to begin at the time point when half distance to the squat height was reached and to end at the time point when half the squat height was exceeded shortly before take-off. The range of the post jump phase was defined to begin at the time point at which half the stabilisation squat height was reached and to end at the time point at which half the stabilisation squat height was exceeded.

For the purpose of this study the Kinect camera was mounted onto a tripod adjusted to a height of $88 \mathrm{~cm}$ and in $290 \mathrm{~cm}$ distance to the center of the force plate on which the athlete was positioned during his jump. The Kinect was moved slightly to the side of the center line in order to free the view for all six VICON cameras $\left(10^{\circ}\right.$ of the VICON center line). No interaction of Kinect with Vicon was observed.

2.5 Vicon and Capture coregistration: The Vicon marker information acquired in the Kinect depth maps was used to coregister the data from the two measurement systems. The highly reflective Vicon markers displayed in the Kinect depth maps as black voids. Hence, each marker could be localised exactly within the Kinect coordinate system. The Kinect data was then rotated and translated for the calibration frame till the Vicon markers detected by Kinect matched the Vicon marker in the Vicon data set. The transformation parameters were extracted and applied to transform the skeletal joint centres.

2.6 Data analysis: The precision and accuracy of measurements was assessed between both systems using Matlab. Vicon data for the three trials were processed using Nexus 2.1.1 and Vicon's Plug in Gait modelling system which calculated joint centers and derived joint kinematics thereafter. Vicon trial data were exported as C3D files which were then used to compare to Kinect data. The raw data was exported to Matlab/Visual 3D for processing. The VICON and Kinect data were coregistered using the location of the VICON markers in MATLAB. The valgus and flexion angles were computed for the knee by using the information provided by the upper and lower leg joint locations (as defined by the hip, knee, and ankle joint coordinates). The valgus angle was computed as the twodimensional angle in the width and height plane. The flexion angle was computed as the two-dimensional angle in the depth and height plane. Please refer to Figure 1 for a graphical representation of this measurement approach. The knee angles were measured for the right and left leg as well as for the pre and post jump phase for each trial. The average and standard deviation was computed for each of these measurements across the number of investigated trials $(\mathrm{N}=3)$.

\section{RESULTS}

Table 1 shows the pre jump phase results for knee valgus and knee flexion, measured in degrees. The refined Kinect data was less accurate at measuring for knee valgus than for knee flexion, the accuracy of the right knee valgus was $13^{\circ}$ while the biggest deviation in accuracy for knee flexion was $2.6^{\circ}$. Table 2 shows the post jump phase results for knee valgus and knee flexion, measured in degrees. Like the pre CMJ values the refined Kinect data showed better accuracy in the measurement of post CMJ knee flexion $\left(-4^{\circ}\right)$ than post CMJ knee valgus $\left(9.9^{\circ}\right)$. Interestingly for both Pre and Post CMJ Kinect measured the right leg more accurately than the left. The display of the anatomical landmarks as seen by the Kinect is shown in Figure 1. Above the graph the point cloud data in side and front view is presented to give an indication of the jumper's pose at the stage of squatting during the CMJ. 


\section{DISCUSSION}

A state-of-the-art biomechanics software was tested in its capability to capture biomechanics information during CMJ. The results showed that, when correction algorithms were applied, knee flexion was measured with high accuracy, deviating less than $4^{\circ}$ from the measured angles using gold standard VICON system. This finding is supported by Stone et al., who also found good accuracy in the measurement of knee flexion. Several studies (Stone et al. 2013; Pfister et al. 2014; Mentiplay et al. 2015) have evaluated the accuracy of the Microsoft Kinect, against gold standard biomechanical analysis tools, for knee flexion during various tasks. Varying degrees of accuracy have been reported for these measurements. Mentiplay et al., (2015) reported a variance in knee flexion angle during walking of up to $30^{\circ}$, similarly (Pfister et al. 2014) reported large errors in knee flexion during walking of up to $10^{\circ}$. The results of the present study show enhanced accuracy of the measurement of knee flexion angles $\left(4^{\circ}\right)$. The reduction in knee flexion error is most likely attributable to the correction algorithms used in order to refine the raw Kinect data. Especially, the bone length correction can have a severe influence on the knee flexion angle as observed in the results of this study. To the best of our knowledge no study has explored the accuracy of the Microsoft Kinect in the measurement of knee valgus in degrees. There was a variance in accuracy between the left and right legs for the valgus measures, there are a number of possible reasons for these differences. One potential reason for this variance could be attributed to the variability of the values of the three CMJs performed by the subject. A second potential reason for the variance could be that infact further improvements in the state-of-the art software are necessary for the Microsoft Kinect. Whilst the Vicon system is seen as the gold standard biomechanical analysis tool, the marker placement is vital to the accuracy of the Vicon and human error in marker placement combined with the movement of the markers during explosive dynamic movements is a concern to the accuracy and repeatability of the Vicon (Stone et al. 2013). These results demonstrate that low-cost markerless motion capture could provide an objective method for assessing lower limb jump and landing mechanics in an applied sports setting with good accuracy and repeatability. Although the results are limited in generalisability due to the single case comparative design, there is sufficient evidence of proof of concept to warrant future research. There are inherent limitations to the present study. A bigger study that examines the measurement method both cross sectionally and longitudinally is necessary to provide more robust evidence base for the accuracy, precision and reliability of the Kinect system over a range of body types and anthropometrics.

\section{CONCLUSION}

The current paper describes initial proof of concept work highlighting the need for more in depth investigations in this area. There is a need for the development of a dynamic movement screening tool that has sufficient sensitivity and specificity for the prediction of future ACL injury risk. The accuracy improvement observed for knee flexion angular measures are promising in terms of developing future algorithms that correct artefacts caused by Kinect. In addition to the applications in sport, more accessible, objective ACL screening has wider implications for physiotherapy and surgical rehabilitation.

\section{REFERENCES}

Blackburn, J. Troy, and Darin A. Padua. 2008. "Influence of Trunk Flexion on Hip and Knee Joint Kinematics during a Controlled Drop Landing." Clinical Biomechanics 23 (3): 313 19.

Boden, B.P., G. S. Dean, J. A. Feagin Jr, and W. E. Garrett Jr. 200. "Mechanisms of Anterior Cruciate Ligament Injury." Orthopedics 23 (6): 573-78.

Bonnechère, Bruno, Bart Jansen, Patrick Salvia, Hakim Bouzahouene, Lubos Omelina, Jan Cornelis, Marcel Rooze, and Serge Van Sint Jan. 2013. "Can the Kinect ${ }^{\mathrm{TM}}$ Sensors Be Used for Motion Analysis?" Transaction on Electrical and Electronic Circuits and Systems 4 (1).

Chappell, Jonathan D., Bing Yu, Donald T. Kirkendall, and William E. Garrett. 2002. "A Comparison of Knee Kinetics between Male and Female Recreational Athletes in Stop-Jump Tasks." The American Journal of Sports Medicine 30 (2): 26167.

Cormie, Prue, Jeffrey M. McBride, and Grant O. McCaulley. 2009. "Power-Time, Force-Time, and Velocity-Time Curve Analysis of the Countermovement Jump: Impact of Training." Journal of Strength and Conditioning Research / National Strength \& Conditioning Association 23 (1): 177-86.

Dufek, J. S., and B. T. Bates. 1991. "Biomechanical Factors Associated with Injury during Landing in Jump Sports." Sports Medicine 12 (5): 326-37.

Faunø, P., and B. Wulff Jakobsen. 2006. "Mechanism of Anterior Cruciate Ligament Injuries in Soccer." International Journal of Sports Medicine 27 (1): 75-79.

Ford, Kevin R., Gregory D. Myer, and Timothy E. Hewett. 2003. "Valgus Knee Motion during Landing in High School Female and Male Basketball Players." Medicine \& Science in Sports \& Exercise 35 (10): 1745-50.

Ford, Kevin R., Gregory D. Myer, Harrison E. Toms, and Timothy E. Hewett. 2005. "Gender Differences in the Kinematics of Unanticipated Cutting in Young Athletes." Medicine and Science in Sports and Exercise 37 (1): 124-29.

Hewett, Timothy E., Gregory D. Myer, Kevin R. Ford, Robert S. Heidt, Angelo J. Colosimo, Scott G. McLean, Antonie J. van den Bogert, Mark V. Paterno, and Paul Succop. 2005a. "Biomechanical Measures of Neuromuscular Control and Valgus Loading of the Knee Predict Anterior Cruciate Ligament Injury Risk in Female Athletes: A Prospective Study." The American Journal of Sports Medicine 33 (4): 492-501.

Hewett, Timothy E., Gregory D. Myer, Kevin R. Ford, Robert S. Heidt Jr, Angelo J. Colosimo, Scott G. McLean, Antonie J. van den Bogert, Mark V. Paterno, and Paul Succop. 2005b. "Biomechanical Measures of Neuromuscular Control and Valgus Loading of the Knee Predict Anterior Cruciate Ligament Injury Risk in Female Athletes: A Prospective Study." The American Journal of Sports Medicine 33 (4): 492-501.

Krosshaug, Tron, Atsuo Nakamae, Barry P. Boden, Lars Engebretsen, Gerald Smith, James R. Slauterbeck, Timothy E. Hewett, and Roald Bahr. 2007. "Mechanisms of Anterior 
Cruciate Ligament Injury in Basketball: Video Analysis of 39 Cases." The American Journal of Sports Medicine 35 (3): 35967.

McLean, Scott G., Susanne W. Lipfert, and Antonie J. van den Bogert. 2004. "Effect of Gender and Defensive Opponent on the Biomechanics of Sidestep Cutting." Medicine and Science in Sports and Exercise 36 (6): 1008-16.

McNair, P. J., R. N. Marshall, and J. A. Matheson. 1990. "Important Features Associated with Acute Anterior Cruciate Ligament Injury." The New Zealand Medical Journal 103 (901): 537-39.

Mentiplay, Benjamin F., Luke G. Perraton, Kelly J. Bower, Yong-Hao Pua, Rebekah McGaw, Sophie Heywood, and Ross A. Clark. 2015. "Gait Assessment Using the Microsoft Xbox One Kinect: Concurrent Validity and Inter-Day Reliability of Spatiotemporal and Kinematic Variables." Journal of Biomechanics 48 (10): 2166-70.

Oñate, James A., Kevin M. Guskiewicz, Stephen W. Marshall, Carol Giuliani, Bing Yu, and William E. Garrett. 2005. "Instruction of Jump-Landing Technique Using Videotape Feedback: Altering Lower Extremity Motion Patterns." The American Journal of Sports Medicine 33 (6): 831-42.

Padua, Darin A., Lindsay J. DiStefano, Anthony I. Beutler, Sarah J. de la Motte, Michael J. DiStefano, and Steven W. Marshall. 2015. "The Landing Error Scoring System as a Screening Tool for an Anterior Cruciate Ligament InjuryPrevention Program in Elite-Youth Soccer Athletes." Journal of Athletic Training 50 (6): 589-95.

Pfister, Alexandra, Alexandre M. West, Shaw Bronner, and Jack Adam Noah. 2014. "Comparative Abilities of Microsoft Kinect and Vicon 3D Motion Capture for Gait Analysis." Journal of Medical Engineering \& Technology 38 (5): 274-80.

Renstrom, P., A. Ljungqvist, E. Arendt, B. Beynnon, T. Fukubayashi, W. Garrett, T. Georgoulis, et al. 2008. "NonContact ACL Injuries in Female Athletes: An International Olympic Committee Current Concepts Statement." British Journal of Sports Medicine 42 (6): 394-412.

Stone, Erik E., Michael Butler, Aaron McRuer, Aaron Gray, Jeffrey Marks, and Marjorie Skubic. 2013. "Evaluation of the Microsoft Kinect for Screening ACL Injury." Conference Proceedings: ... Annual International Conference of the IEEE Engineering in Medicine and Biology Society. IEEE Engineering in Medicine and Biology Society. Conference 2013: 4152-55.

Swanik, Charles Buz, Tracey Covassin, David J. Stearne, and Philip Schatz. 2007. "The Relationship between Neurocognitive Function and Noncontact Anterior Cruciate Ligament Injuries." The American Journal of Sports Medicine 35 (6): 943-48.

Zeller, Brian L., Jean L. McCrory, W. Ben Kibler, and Timothy L. Uhl. 2003. "Differences in Kinematics and Electromyographic Activity between Men and Women during the Single-Legged Squat." The American Journal of Sports Medicine 31 (3): 449-56.

\section{APPENDIX}

FIGURE 1.
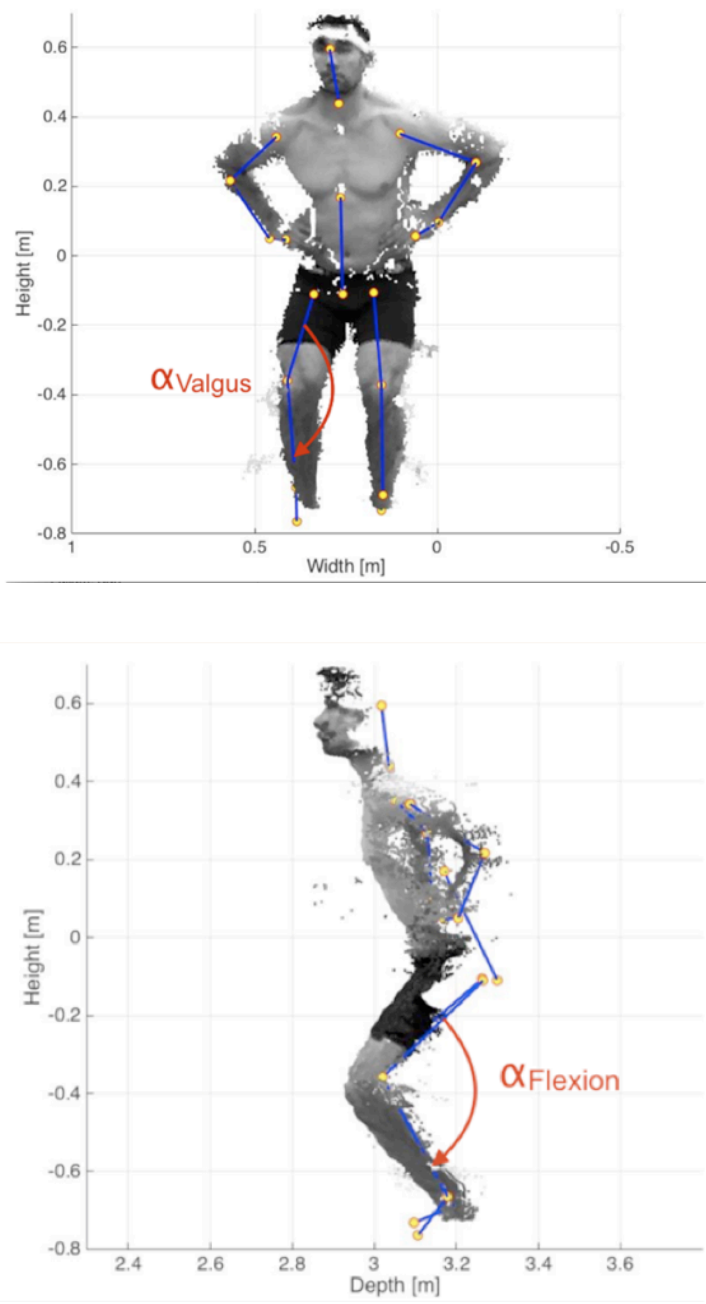

TABLE 1.

\begin{tabular}{|c|c|c|c|c|c|}
\hline Pre Jump & & \multicolumn{2}{|c|}{ Left } & \multicolumn{2}{|c|}{ Right } \\
\hline & & $\begin{array}{c}\text { Pre } \\
\text { Vicon }\end{array}$ & $\begin{array}{c}\text { Pre } \\
\text { Kinect }\end{array}$ & $\begin{array}{c}\text { Pre } \\
\text { Vicon }\end{array}$ & $\begin{array}{c}\text { Pre } \\
\text { Kinect }\end{array}$ \\
\hline Valgus $\left[{ }^{\circ}\right]$ & CMJ 1 & 24.9 & 35.1 & 3.3 & -1.4 \\
\hline & CMJ 2 & 17.1 & 33.2 & 4.3 & 0.1 \\
\hline & CMJ 3 & 9.8 & 29.5 & 6.8 & -0.4 \\
\hline & $\begin{array}{c}\text { Mean } \\
\pm \\
\text { std }\end{array}$ & $\begin{array}{l}17.2 \\
\pm \\
7.6\end{array}$ & $\begin{array}{c}32.6 \\
\pm \\
2.2\end{array}$ & $\begin{array}{c}4.8 \\
\pm \\
1.8\end{array}$ & $\begin{array}{c}-0.6 \\
\pm \\
0.8\end{array}$ \\
\hline & $\begin{array}{c}\text { Accuracy } \\
\pm \\
\text { Precision }\end{array}$ & \multicolumn{2}{|c|}{$13 \pm 5.5$} & \multicolumn{2}{|c|}{$-5.4 \pm 1.6$} \\
\hline \multirow[t]{5}{*}{$\begin{array}{c}\text { Knee } \\
\text { flexion }\left[{ }^{\circ}\right]\end{array}$} & CMJ 1 & 79.3 & 74 & 74.2 & 77.2 \\
\hline & CMJ2 & 74.9 & 73.8 & 72.5 & 74.4 \\
\hline & CMJ3 & 78.3 & 80.2 & 76.6 & 79.7 \\
\hline & $\begin{array}{c}\text { Mean } \\
\quad \pm \\
\text { std }\end{array}$ & $\begin{array}{c}77.5 \\
\pm \\
2.3 \\
\end{array}$ & $\begin{array}{c}76 \\
\pm \\
3.6\end{array}$ & $\begin{array}{c}74.4 \\
\pm \\
2 \\
\end{array}$ & $\begin{array}{c}77.1 \\
\pm \\
2.6\end{array}$ \\
\hline & $\begin{array}{c}\text { Accuracy } \\
\pm \\
\text { Precision }\end{array}$ & \multicolumn{2}{|c|}{$-1.5 \pm 3.6$} & \multicolumn{2}{|c|}{$2.6 \pm 0.7$} \\
\hline
\end{tabular}


The International Archives of the Photogrammetry, Remote Sensing and Spatial Information Sciences, Volume XL-5/W8, 2016 LowCost3D (LC3D), Sensors, Algorithms, Applications, 1-2 December 2015, Berlin, Germany

TABLE 2

\begin{tabular}{|c|c|c|c|c|c|}
\hline Post Jump & & \multicolumn{2}{|c|}{ Left } & \multicolumn{2}{|c|}{ Right } \\
\hline & & $\begin{array}{l}\text { Post } \\
\text { Vicon }\end{array}$ & $\begin{array}{c}\text { Post } \\
\text { Kinect }\end{array}$ & $\begin{array}{l}\text { Post } \\
\text { Vicon }\end{array}$ & $\begin{array}{c}\text { Post } \\
\text { Kinect }\end{array}$ \\
\hline Valgus $\left[{ }^{\circ}\right]$ & CMJ 1 & 27.2 & 32.8 & 7 & 1.1 \\
\hline & CMJ 2 & 17.3 & 29.9 & 4.9 & -0.1 \\
\hline & CMJ 3 & 10 & 21.7 & 8.6 & 0.7 \\
\hline & $\begin{array}{c}\text { Mean } \\
\pm \\
\text { std }\end{array}$ & $\begin{array}{l}18.2 \\
\pm \\
8.6\end{array}$ & $\begin{array}{c}281 . \\
\pm \\
5.8\end{array}$ & $\begin{array}{c}6.9 \\
\pm \\
1.9\end{array}$ & $\begin{array}{c}0.6 \\
\pm \\
1.5\end{array}$ \\
\hline & $\begin{array}{c}\text { Accuracy } \\
\pm \\
\text { Precision } \\
\end{array}$ & \multicolumn{2}{|c|}{$9.9 \pm 3.8$} & \multicolumn{2}{|c|}{$-6.3 \pm 1.5$} \\
\hline \multirow[t]{5}{*}{$\begin{array}{c}\text { Knee } \\
\text { flexion }\left[{ }^{\circ}\right]\end{array}$} & CMJ 1 & 78.6 & 71.7 & 71.1 & 75.9 \\
\hline & CMJ2 & 69.8 & 68.9 & 68.6 & 69.1 \\
\hline & CMJ3 & 72.4 & 68.9 & 68.2 & 67.9 \\
\hline & $\begin{array}{c}\text { Mean } \\
\pm \\
\text { std }\end{array}$ & $\begin{array}{l}73.6 \\
\pm \\
4.5\end{array}$ & $\begin{array}{c}69.8 \\
\pm \\
1.6\end{array}$ & $\begin{array}{c}69.3 \\
\pm \\
1.5\end{array}$ & $\begin{array}{c}71 \\
\pm \\
4.3\end{array}$ \\
\hline & $\begin{array}{c}\text { Accuracy } \\
\pm \\
\text { Precision } \\
\end{array}$ & \multicolumn{2}{|c|}{$-3.8 \pm 3$} & \multicolumn{2}{|c|}{$1.7 \pm 2.8$} \\
\hline
\end{tabular}

